\title{
Mucormycosis in two community hospitals and the role of infectious disease consultation: a case series
}

This article was published in the following Dove Press journal:

International Journal of General Medicine

29 October 2013

Number of times this article has been viewed

\author{
Yue Dail \\ James W Walker' \\ Ruba A Halloush ${ }^{2}$ \\ Faisal A Khasawneh ${ }^{3}$ \\ 'Department of Internal Medicine, \\ Texas Tech University Health Sciences \\ Center, ${ }^{2}$ Amarillo Pathology Group, \\ ${ }^{3}$ Section of Infectious Diseases. \\ Department of Internal Medicine, \\ Texas Tech University Health Sciences \\ Center, Amarillo, TX, USA
}

Background: Mucorales are ubiquitous filamentous fungi that can cause a devastating, invasive infection. This order has become an increasingly important pathogen during the last two decades, due to the dramatic increase in patients with predisposing factors. The aim of this retrospective study was to report the clinical characteristics, therapeutic options, and outcomes of patients diagnosed with mucormycosis in community hospitals in Amarillo, Texas, and to reflect on the role of infectious disease (ID) physicians in managing this potentially lifethreatening problem.

Patients and methods: This was a retrospective chart review of patients hospitalized with mucormycosis in two community hospitals in Amarillo between January 1, 2001 and December 31, 2011.

Results: Ten patients were diagnosed with mucormycosis during the study period, with a mean age of 58.8 years. There were five cases of pulmonary infection, two cases of cutaneous infection, two cases of rhinocerebral infection, and one case of gastrointestinal infection. Poorly controlled diabetes was the most common risk factor, identified in six patients, followed by hematological malignancy, immunosuppression, and trauma. ID physicians were consulted in all cases, albeit late in some cases. Nine patients received antifungal therapy, and five patients received surgical debridement. Lipid formulations of amphotericin B were prescribed for eight patients, used alone in two cases, and combined with caspofungin and posaconazole in one and five cases, respectively. One patient was treated with posaconazole alone. Eight patients were discharged from the hospital alive. The mortality rate at 6 -month follow-up was $40 \%$.

Conclusion: Mucormycosis is an emerging fungal infection that continues to carry significant morbidity and mortality. At-risk patient populations are on the rise, and include those with poorly controlled diabetes mellitus. Early diagnosis, in consultation with an ID physician, and an aggressive combined approach with surgical debridement and combined antifungal therapy is pivotal in improving patients' outcomes.

Keywords: mucormycosis, treatment, outcome, community hospitals

\section{Introduction}

Mucorales are ubiquitous filamentous fungi that can cause a life-threatening infection in immunocompromised patients. ${ }^{1}$ Predisposing conditions include diabetes mellitus, malignant hematological diseases, stem cell and organ transplant, neutropenia, iron overload, major trauma, immunosuppressive medications, human immunodeficiency virus infection, and chronic liver disease. The hallmark of this infection is tissue necrosis resulting from angioinvasion and subsequent thrombosis, with substantial associated morbidity and mortality. ${ }^{2}$ \footnotetext{
Section of Infectious Diseases, Department of Internal Medicine, Texas Tech University Health Sciences Center, I 400 South Coulter Street, Amarillo, TX 79106, USA

$\mathrm{Tel}+\mathrm{I} 8063545480$

Fax +I 8063545765

Email faisal.khasawneh@ttuhsc.edu
}

submit your manuscript | www.dovepress.com

Dovepress

http://dx.doi.org// 0.2147//JGM.S52718 
Based on anatomic location, mucormycosis can cause one of six forms of infection: rhinocerebral, pulmonary, cutaneous, gastrointestinal, disseminated, and uncommon presentations, including endocarditis, osteomyelitis, and peritonitis. ${ }^{1}$ Irrespective of the location, the infection is rapidly progressive and results in death unless the underlying risk factors are corrected and aggressive treatment with antifungal agents and surgical debridement is instituted.

The aim of this retrospective study was to report the clinical characteristics, therapeutic options, and outcomes of patients diagnosed with mucormycosis in community hospitals in Amarillo, Texas, and to explore the role of infectious disease (ID) consultation in establishing diagnosis and treating this life-threatening infection.

\section{Patients and methods}

This was a retrospective chart-review study conducted at Northwest Texas Hospital and Baptist Saint Anthony's Hospital, the only two community hospitals in Amarillo, Texas, between January 1, 2001 and December 31, 2011. Both hospitals are teaching hospitals for Texas Tech University Health Sciences Center (TTUHSC) and serve a large catchment area in West Texas. The study was approved by the institutional review committees at TTUHSC and both hospitals. The medical records and pathology archives of patients admitted to these facilities during the study period were queried for a diagnosis of mucormycosis, International Classification of Diseases (ICD)-9 code 117.7. Cases that were diagnosed on an outpatient basis or in day surgery and were not admitted to either hospital were excluded. The charts of identified patients were reviewed for the following information: demographics, predisposing conditions, anatomical location of infection, time of ID consultation and its contribution to management, therapy including surgical interventions and antifungal drugs, therapeutic side effects, length of hospital stay, vital status and disposition at hospital discharge, and vital status at 6-month follow-up.

Mucormycosis cases were classified into one of the following categories:

1. Proven infection: if there was a histopathological documentation of mucormycosis and a positive culture result from a normally sterile site

2. Probable infection: if there was a histopathological or microbiological documentation of mucormycosis in a susceptible host with consistent clinical features

3. Possible infection: if there was a histopathological or microbiological documentation of mucormycosis or consistent clinical features in a susceptible host.

\section{Statistical analysis}

For continuous variables, mean values with range are presented. For each categorical variable, the number of patients with that variable and a corresponding percentage are reported.

\section{Results}

During the study period, 14 patients were diagnosed with mucormycosis. All were confirmed by histopathological exam; however, none had a positive microbiological culture, and therefore the exact species was not determined. Four of the 14 patients had paranasal mucormycosis sinusitis. These patients underwent day surgeries and were treated as outpatients, and therefore were excluded from further analysis. The remaining ten patients - seven males and three females - were diagnosed with mucormycosis and required hospitalization. Diabetes was the most common predisposing condition, identified in six patients. Of these, five had diabetes as the only risk factor recognized. Table 1 summarizes the ten patients' demographics, presentation, risk factors, and outcome. As seen in Table 1, three patients had neutropenia on admission. The mean white blood cell count for the other seven patients was $12.9 \times 10^{9} / \mu \mathrm{L}$ (range 6.3-23.1). Examples of patients' computed tomography scan results and histopathology are shown in Figures 1-3.

All of the described cases were managed in consultation with four different ID specialists. In patients with gastrointestinal mucormycosis, cutaneous mucormycosis, and one case of rhinocerebral infection - a total of four cases - the consultation was requested after the pathology report demonstrated findings consistent with mucormycosis. In three of these cases, no fungal cultures were sent; meanwhile, fungal cultures were sent on a subsequent debridement session from the patient with rhinocerebral infection. In the six other cases, ID consultation was conducted earlier, which helped to guide workup targeting a broader spectrum of potential pathogens.

Antifungal agents were given in standard doses: liposomal amphotericin B and amphotericin B lipid complex were dosed intravenously at $5 \mathrm{mg} / \mathrm{kg}$, amphotericin B deoxycholate was dosed intravenously at $1 \mathrm{mg} / \mathrm{kg}$, posaconazole was dosed orally at $400 \mathrm{mg}$ given twice daily, and caspofungin was dosed intravenously at $100 \mathrm{mg}$ daily. Patients received the intravenous agents throughout their hospitalization, and received posaconazole for several months afterwards. The fact that treatment was given at different settings, including long-term acute-care hospitals and the outpatient setting, made it difficult to ascertain the exact duration of therapy.

The most common side effect of treatment was acute kidney injury, and it was reported in four patients. The renal 
Table I The characteristics of ten patients with mucormycosis in two community hospitals in West Texas

\begin{tabular}{|c|c|}
\hline Patient & Salient features of ten cases of mucormycosis \\
\hline I & $\begin{array}{l}\text { 29-yo Asian } M \text { admitted with newly diagnosed lupus nephritis and pneumonitis. He was treated with cyclophosphamide and } \\
\text { methylprednisolone. Hospital course complicated by hospital-acquired pneumonia needing lengthy course of abx and later on } \\
\text { progressive necrotizing pneumonia. Open-lung biopsy was consistent with pulmonary mucormycosis. Liposomal amphotericin B } \\
\text { and posaconazole initiated, but patient died on the 30th hospital day. }\end{array}$ \\
\hline 2 & $\begin{array}{l}\text { 54-yo W M admitted unconscious in DKA. He had a necrotic skin patch over the left cheek. CT head was negative. Poorly } \\
\text { controlled DM }\left(\mathrm{HbA}_{\mathrm{Ic}} \text { I4\%) was managed, bacterial infections ruled out, and the necrotic area was treated as a pressure injury }\right. \\
\text { related to his presentation. The patient was kept in the hospital pending placement when he had new-onset seizures during the } \\
\text { second week of hospitalization. Head imaging showed a progressive invasive process involving the left maxillary sinus and frontal } \\
\text { lobe. Antibiotics, including liposomal amphotericin B and posaconazole, were initiated. Multiple debridement sessions were } \\
\text { done. Pathology findings were consistent with rhinocerebral mucormycosis. The patient was discharged alive after } 126 \text { days' } \\
\text { hospitalization to an } \mathrm{NH} \text {. Patient was still alive at } 6 \text {-month follow-up. }\end{array}$ \\
\hline 3 & $\begin{array}{l}\text { 60-yo AA M admitted with poorly controlled } \mathrm{DM}\left(\mathrm{HbA}_{\mathrm{Ic}} \mathrm{II.7 \% )} \text {, subacute right facial swelling, and tooth pain. Patient failed }\right. \\
\text { oral antibiotics given for a suspected periodontal infection. } \mathrm{CT} \text { sinuses showed a destructive process involving the } \mathrm{R} \text { maxillary } \\
\text { sinus. OR surgical exploration and debridement as well as biopsies were consistent with rhinocerebral mucormycosis. Liposomal } \\
\text { amphotericin } \mathrm{B} \text { and posaconazole were initiated. Patient was discharged alive after } 20 \text { days' hospitalization to an LTAC. He was } \\
\text { alive at 6-month follow-up. }\end{array}$ \\
\hline 4 & $\begin{array}{l}55 \text {-yo W M admitted with subacute necrotizing pneumonia. The patient was found to be neutropenic }\left(\mathrm{WBC} 2.6 \times 10^{9} / \mu \mathrm{L}\right) \text { due to } \\
\text { methimazole. Given the poor response to broad-spectrum antibiotics, he underwent CT-guided biopsy. The pathology findings } \\
\text { were consistent with pulmonary mucormycosis. WBC improved off methimazole. Posaconazole was initiated. Patient was } \\
\text { discharged home after } 13 \text { days' hospitalization. He was alive at } 6 \text {-month follow-up. }\end{array}$ \\
\hline 5 & $\begin{array}{l}\left.\text { 59-yo W M with relapsed peripheral T-cell lymphoma and DM admitted with neutropenic (WBC } 1.5 \times 10^{9} / \mu \mathrm{L}\right) \text { fever. CT chest } \\
\text { showed b/l pulmonary nodules. CT-guided biopsy was consistent with pulmonary mucormycosis. Amphotericin B lipid complex } \\
\text { was initiated. Patient was discharged home after } 23 \text { days' hospitalization. Follow-up CT chest showed regression of nodules. The } \\
\text { patient died } 3 \text { months later due to lymphoma progression. }\end{array}$ \\
\hline 6 & $\begin{array}{l}\text { 79-yo W M involved in a road traffic accident with trauma to the } L \text { leg. He developed a necrotizing skin infection that did not } \\
\text { respond to broad-spectrum antibiotics and surgical debridement. The patient underwent AKA. Deep surgical samples were } \\
\text { consistent with cutaneous mucormycosis. The surgical margin did not show any involvement. No antifungals were given. Patient } \\
\text { was discharged home after II days' hospitalization and was alive at 6-month follow-up. }\end{array}$ \\
\hline 7 & $\begin{array}{l}\text { 47-yo W F found unconscious. She was treated for aspiration pneumonia and anoxic brain injury. Her poorly controlled DM } \\
\left(\mathrm{HbA}_{\mathrm{IC}} \text { I3.2\%) was managed. She never regained consciousness. She had a lengthy ICU course with multiple complications. She }\right. \\
\text { developed progressive cavitary pneumonia that did not respond to broad-spectrum antibiotics. CT-guided biopsy was consistent } \\
\text { with pulmonary mucormycosis. Amphotericin B lipid complex was initiated, but she died on day } 196 \text { of hospitalization. }\end{array}$ \\
\hline 8 & $\begin{array}{l}\left.\text { 67-yo W F with AML admitted with neutropenia (WBC } 3.2 \times 10^{9} / \mu \mathrm{L}\right) \text {, dry cough, and R-sided pleuritic chest pain. CT chest showed } \\
\text { a mass lesion that was biopsied, with findings consistent with pulmonary mucormycosis. Liposomal amphotericin B and caspofungin } \\
\text { were initiated. Patient was discharged home on day } 7 \text { of hospitalization. Follow-up CT chest showed regression of mass. She died } \\
\text { of her leukemia after } 5 \text { months. }\end{array}$ \\
\hline 9 & $\begin{array}{l}\text { 6I-yo W M admitted with progressive necrotizing skin infection to the } L \text { knee that developed a few days after he sustained a } \\
\text { trauma. Poorly controlled DM }\left(\mathrm{HbA}_{\mathrm{Ic}} 9.8 \%\right) \text { was managed. He failed broad-spectrum antibiotics and debridement. He underwent } \\
\text { AKA. Surgical margins showed fungal hyphae consistent with cutaneous mucormycosis. Liposomal amphotericin } B \text {, posaconazole, } \\
\text { and deferasirox were initiated. He was discharged to an LTAC. He was alive at 6-month follow-up. }\end{array}$ \\
\hline 10 & 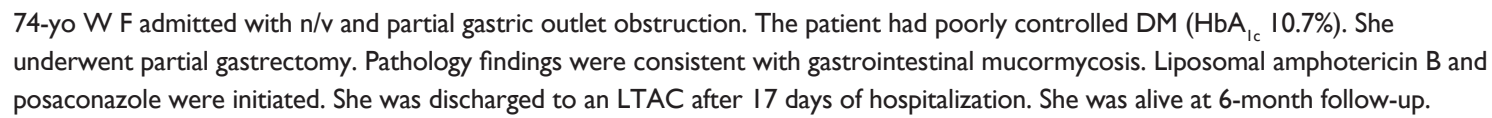 \\
\hline
\end{tabular}

Abbreviations: yo, year-old; M, male; abx, antibiotics; W, white; DKA, diabetic ketoacidosis; DM, diabetes mellitus; CT, computed tomography; HbA ${ }_{I C}$, hemoglobin $A_{I C}$; AA, African American; R, right; OR, operating room; LTAC, long-term acute-care hospital; WBC, white blood cell count; b/l, bilateral; L, left; AKA, above-knee amputation; $\mathrm{F}$, female; ICU, intensive care unit; $\mathrm{AML}$, acute myeloid leukemia; $\mathrm{NH}$, nursing home; $\mathrm{n} / \mathrm{v}$, nausea and vomiting.

insult was managed by intravenous hydration with normal saline, discontinuing other nephrotoxic medications, and administering amphotericin $\mathrm{B}$ at prolonged dosing intervals of 48-72 hours. One patient developed an infusion reaction to amphotericin B lipid complex, characterized by fever and rigors, and was managed symptomatically.

Eight patients were discharged from the hospital alive; four were released home and four transferred to long-term care facilities. Four patients required intensive care-unit stay, with mean duration of 62 days (range 5-196 days). The cohort mean hospital length of stay was 46.1 days (range 7-196 days). All-cause mortality rate at 6-month follow-up was $40 \%$.

\section{Discussion}

Mucormycosis is a devastating infection with high morbidity and mortality. ${ }^{3}$ Ten histopathologically confirmed cases 


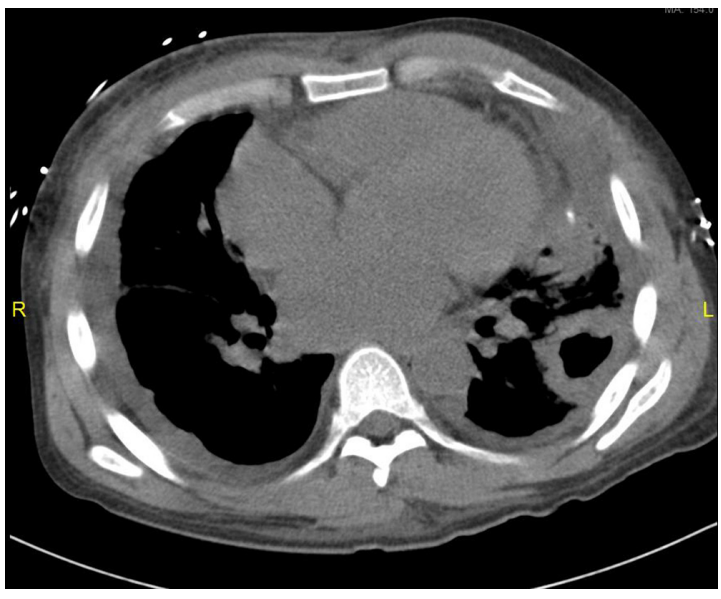

Figure I Computed tomography scan section of patient number I chest, showing a cavitary left-sided lung mass.

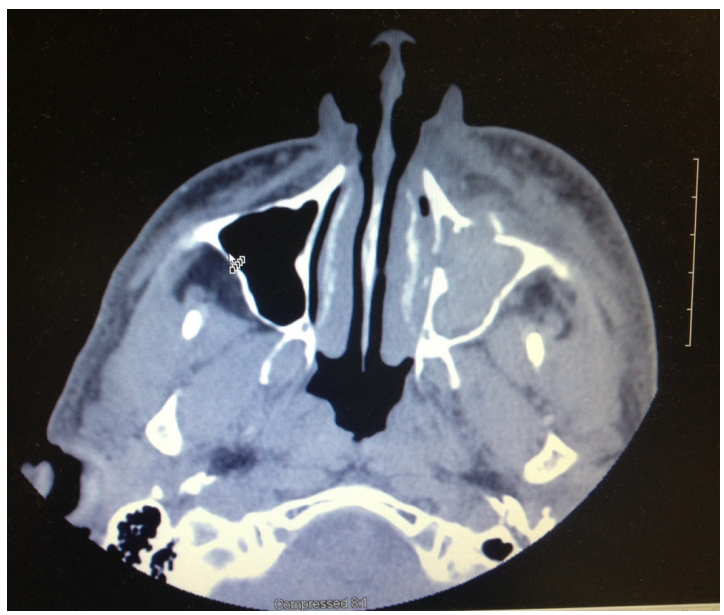

Figure 2 Computed tomography scan of the maxillary sinuses of patient number 3 , showing a soft-tissue mass in the left maxillary sinus with bony destruction of the anterior wall of the sinus.

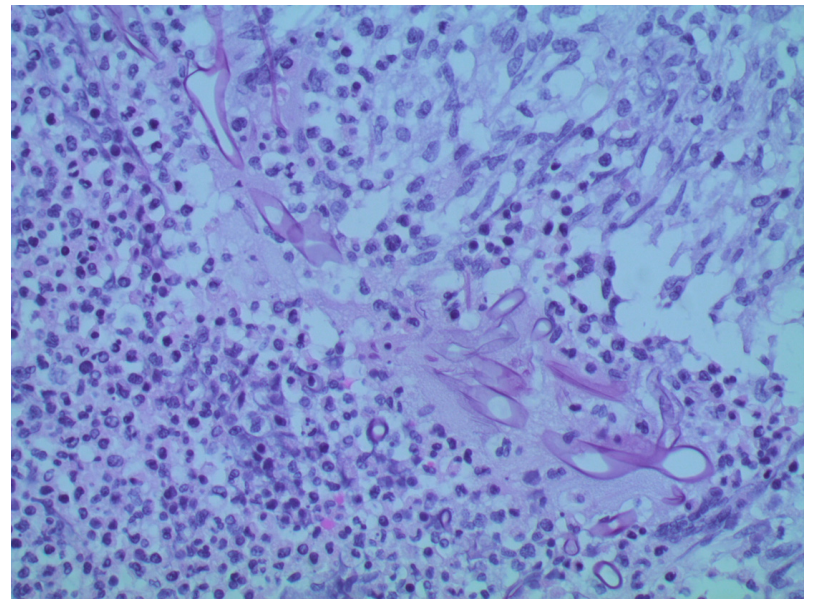

Figure 3 Hematoxylin and eosin-stained histopathology section from patient number 9 amputated leg soft-tissue margin, showing broad, pauciseptate fungal hyphae, consistent with mucormycosis, in a background of necrotizing granulomatous inflammation (original magnification 400x). were diagnosed in Amarillo, Texas between January 1, 2001 and December 31, 2011. Our study describes five pulmonary infections, two cutaneous infections, two cases of rhinocerebral infection, and one case of gastrointestinal infection. Predisposing conditions included diabetes mellitus, hematologic malignancy, immunosuppression, and trauma. Treatment modalities included antifungal drugs and surgical debridement. An ID physician was consulted in every case, albeit late in some cases, which explains why fungal cultures were not sent in those cases. A combination of two drugs or more was used in 50\% of cases. The infection and its complications claimed the lives of two patients, with an overall mortality rate of $40 \%$ at 6 months.

"Zygomycosis" was classically used to describe infections caused by species in the orders Mucorales and Entomophthorales. ${ }^{4}$ Evolving changes in nomenclature and molecular taxonomy favors the use of "mucormycosis" over the inclusive name "zygomycosis" to describe these two clinicopathologically different infections. Hence, we used the term "mucormycosis" exclusively throughout our paper.

Epidemiological studies of mucormycosis are difficult and complex, because the disease is rare and lacks mass surveillance. Therefore, larger analyses typically rely on reviewing multiple smaller case series. ${ }^{3,5}$ Published mucormycosis case series from community hospitals in the US during the past two decades have been scant. With a time frame between January 1, 2001 and December 31, 2011, we have reported our experience with cases of mucormycosis at two community hospitals in West Texas.

Despite improvements in imaging modalities leading to earlier diagnoses and increasing therapeutic options, mucormycosis is still difficult to treat. In a retrospective study of 929 mucormycosis cases, Roden et al reported improving patient survival over the last half-century, especially in patients treated with antifungal therapy and surgery. ${ }^{3}$ The survival rate in that group was $70 \% .{ }^{3}$ Likewise, in our cohort, the relatively low 6-month mortality rate of $40 \%$ can be attributed to the high number of patients with potentially reversible risk factors and the low number of profoundly immunocompromised hosts, namely transplant patients and patients with hematological malignancy. Two patients with aggressive pulmonary infection died as inpatients and two died later on from their underlying malignancies, despite documented improvement in their fungal infection - pulmonary lesion shrinkage on chest computed tomography.

Although amphotericin B deoxycholate is the only antifungal agent licensed by the US Food and Drug 
Administration for primary therapy of mucormycosis, most of our patients received its lipid formulations. ${ }^{6}$ This is due to the lower toxicity associated with amphotericin B lipid formulations and better efficacy reported in animal models and some retrospective clinical studies. ${ }^{7,8}$

More recent studies show higher mortality with antifungal monotherapy despite standard dosing, especially in hematological patients. ${ }^{9}$ Though data are limited and the hematological malignancies group is at a disadvantage to start with given their severe immunocompromised state, there has been a growing trend toward combination therapy, and this is demonstrated in our data. The combination of polyene and echinocandin has the upper hand. It has shown synergy and improved survival in animal studies, as well as a small, retrospective clinical study. ${ }^{10,11}$ On the contrary, posaconazolecontaining regimens are plagued by significant variability in serum levels and poor activity against some of the most common fungal species that cause mucormycosis. ${ }^{12,13}$

Despite that argument, five of our ten patients received a polyene-posaconazole combination. Of these patients, four $(80 \%)$ survived. Furthermore, one patient was successfully treated with posaconazole alone. On the other hand, only one of our patients was treated with a polyene-echinocandin regimen. This patient's infection responded well to therapy; however, he ultimately succumbed to complications of his underlying malignancy. We speculate that the former regimen was favored in our case series because posaconazole is an oral agent that was intended to be used to continue therapy in the outpatient setting and because the positive results achieved encouraged further use.

One patient in our cohort received the iron chelator deferasirox alongside liposomal amphotericin B and posaconazole. The patient had a favorable outcome. Deferasirox's cost and limited supporting data curbed wider use. A double-blinded, randomized, placebo-controlled trial failed to demonstrate a benefit of combining deferasirox with liposomal amphotericin B against mucormycosis in a heterogeneous group of patients..$^{14}$

ID consultation is most appropriate in handling resistant infections, atypical or uncommon infections, as well as infections in immunocompromised hosts, and infections that are failing first-line therapy. In our cohort, an ID physician directed antifungal therapy and follow-up, but in almost half the cases, consultation was placed after the histopathology came back consistent with mucormycosis. This arguable delay in consultation did not compromise outcome. However, earlier consultation would have facilitated making definite diagnosis of fungal infections and other atypical infections, such as mycobacterial infections, if present, by the physician's ordering of appropriate microbiological testing.

This study has several limitations, not the least of which is the small sample size. Furthermore, the retrospective nature limited the amount and accuracy of the information that could be collected. Moreover, the lack of positive microbiological cultures and the limited availability of molecular diagnostic methods made them probable mucormycosis cases. ${ }^{15}$

In conclusion, mucormycosis has been on the rise due to increasing numbers of patients with predisposing conditions. Alongside different immunomodulating disease processes and therapies, diabetes mellitus is an important risk factor for mucormycosis in community hospitals. Currently recommended therapies carry significant toxicity. Many combination therapies have been proposed, researched, and are being used in clinical practice, but indications for multidrug regimens have not been well established. Therefore, potential for future studies on the efficacy of multidrug therapy for mucormycosis remains. Consulting an ID physician when faced with necrotizing or cavitary infections and infections that fail to respond to appropriate first-line antibiotic therapy, especially in immunocompromised hosts and poorly controlled diabetics, should be considered.

\section{Disclosure}

The authors report no conflicts of interest in this work.

\section{References}

1. Petrikkos G, Skiada A, Lortholary O, Roilides E, Walsh TJ, Kontoyiannis DP. Epidemiology and clinical manifestations of mucormycosis. Clin Infect Dis. 2012;54 Suppl 1:S23-S34.

2. Ibrahim AS, Spellberg B, Walsh TJ, Kontoyiannis DP. Pathogenesis of mucormycosis. Clin Infect Dis. 2012;54 Suppl 1:S16-S22.

3. Roden MM, Zaoutis TE, Buchanan WL, et al. Epidemiology and outcome of zygomycosis: a review of 929 reported cases. Clin Infect Dis. 2005;41(5):634-653.

4. Kwon-Chung KJ. Taxonomy of fungi causing mucormycosis and entomophthoramycosis (zygomycosis) and nomenclature of the disease: molecular mycologic perspectives. Clin Infect Dis. 2012;54 Suppl 1: S8-S15.

5. Lanternier F, Dannaoui E, Morizot G, et al. A global analysis of mucormycosis in France: the RetroZygo Study (2005-2007). Clin Infect Dis. 2012;54 Suppl 1:S35-S43.

6. Spellberg B, Ibrahim A, Roilides E, et al. Combination therapy for mucormycosis: why, what, and how? Clin Infect Dis. 2012;54 Suppl 1: S73-S78.

7. Ibrahim AS, Avanessian V, Spellberg B, Edwards JE Jr. Liposomal amphotericin B, and not amphotericin B deoxycholate, improves survival of diabetic mice infected with Rhizopus oryzae. Antimicrob Agents Chemother. 2003;47(10):3343-3344.

8. Moreau P, Milpied N, Fayette N, Ramee JF, Harousseau JL. Reduced renal toxicity and improved clinical tolerance of amphotericin $\mathrm{B}$ mixed with intralipid compared with conventional amphotericin B in neutropenic patients. J Antimicrob Chemother. 1992;30(4):535-541. 
9. Spellberg B, Edwards J Jr, Ibrahim A. Novel perspectives on mucormycosis: pathophysiology, presentation, and management. Clin Microbiol Rev. 2005;18(3):556-569.

10. Reed C, Bryant R, Ibrahim AS, et al. Combination polyene-caspofungin treatment of rhino-orbital-cerebral mucormycosis. Clin Infect Dis. 2008;47(3):364-371.

11. Ibrahim AS, Gebremariam T, Fu Y, Edwards JE Jr, Spellberg B. Combination echinocandin-polyene treatment of murine mucormycosis. Antimicrob Agents Chemother. 2008;52(4):1556-1558.

12. Dannaoui E, Meis JF, Loebenberg D, Verweij PE. Activity of posaconazole in treatment of experimental disseminated zygomycosis. Antimicrob Agents Chemother. 2003;47(11):3647-3650.

13. Krishna G, Sansone-Parsons A, Martinho M, Kantesaria B, Pedicone L. Posaconazole plasma concentrations in juvenile patients with invasive fungal infection. Antimicrob Agents Chemother. 2007;51(3): 812-818.
14. Spellberg B, Ibrahim AS, Chin-Hong PV, et al. The DeferasiroxAmBisome Therapy for Mucormycosis (DEFEAT Mucor) study: a randomized, double-blinded, placebo-controlled trial. J Antimicrob Chemother. 2012;67(3):715-722.

15. De Pauw B, Walsh TJ, Donnelly JP, et al. Revised definitions of invasive fungal disease from the European Organization for Research and Treatment of Cancer/Invasive Fungal Infections Cooperative Group and the National Institute of Allergy and Infectious Diseases Mycoses Study Group (EORTC/MSG) Consensus Group. Clin Infect Dis. 2008;46(12):1813-1821.

\section{Publish your work in this journal}

The International Journal of General Medicine is an international, peer-reviewed open-access journal that focuses on general and internal medicine, pathogenesis, epidemiology, diagnosis, monitoring and treatment protocols. The journal is characterized by the rapid reporting of reviews, original research and clinical studies across all disease areas.
A key focus is the elucidation of disease processes and management protocols resulting in improved outcomes for the patient. The manuscript management system is completely online and includes a very quick and fair peer-review system. Visit http://www.dovepress.com/ testimonials.php to read real quotes from published authors.

Submit your manuscript here: http://www.dovepress.com/international-journal-of-general-medicine-journal 